\title{
A PLASMA CHANNEL BEAM CONDITIONER FOR A FREE ELECTRON LASER*
}

\author{
G. Penn ${ }^{\dagger}$, A.M. Sessler, J.S. Wurtele ${ }^{\ddagger}$, \\ Lawrence Berkeley National Laboratory, Berkeley, CA 94720, USA
}

\begin{abstract}
By "conditioning" an electron beam, through establishing a correlation between transverse action and energy within the beam, the performance of free electron lasers (FELs) can be dramatically improved. Under certain conditions, the FEL can perform as if the transverse emittances of the beam were substantially lower than the actual values. After a brief review of the benefits of beam conditioning, we present a method to generate this correlation through the use of a plasma channel. The strong transverse focusing produced by a plasma channel (chosen to have density $10^{16} / \mathrm{cm}^{3}$ ) allows the optimal correlation to be achieved in a reasonable length channel, of order $1 \mathrm{~m}$. This appears to be a convenient and practical method for achieving conditioned beams, in comparison with other methods which require either a long beamline or multiple passes through some type of ring.
\end{abstract}

\section{INTRODUCTION}

Beam conditioning, that is, the introduction of a correlation between electron transverse action and energy, has been shown to be advantageous for free electron laser (FEL) performance [1]. The concept was introduced 15 years ago, and there has been considerable effort to design a convenient "conditioner". These efforts have greatly clarified the subject, and produced some methods for conditioning, but have not produced a method that is suitable for implementation with current technology and at a reasonable cost $[2,3,4]$. A more recent proposal for a conditioner involves manipulating the electron beam within magnetic wigglers using lasers [5].

There is a strong motivation to continue the search for novel methods for beam conditioning. In this paper, we introduce the concept of a conditioner employing a plasma channel. We concede that a plasma device will add complexity to accelerator operation. On the other hand, great progress has been made in producing plasma channels for accelerating particles and the device proposed here is comparable to what has already been achieved [6]. We propose a plasma conditioner that works on a similar principle to the conditioners already proposed, but because the focusing strength and accelerating gradients achievable in a plasma greatly exceed that of conventional components the

\footnotetext{
* This work was supported by the Director, Office of Science, High Energy Physics, U.S. Department of Energy under Contract No. DE-AC02$05 \mathrm{CH} 11231$.

$\dagger$ gepenn@lbl.gov

$\ddagger$ Also at University of California, Berkeley, Berkeley, CA 94720, USA
}

proposed device is modest in size and appears to be worthy of serious consideration.

In this paper, we give examples of conditioners that might be employed at the Linac Coherent Light Source (LCLS) [7]. One example has the conditioning performed at a beam energy of $100 \mathrm{MeV}$, after which the beam must be compressed and accelerated to the nominal parameters without losing its conditioning. We show that even if the emittance were a factor of two larger than that designed, with a conditioned beam the FEL would still work to design. A second example conditions a beam at $1 \mathrm{GeV}$ beam energy, employing both plasma focusing and plasma acceleration, but further beam compression is not required. The resulting beams are then used in FEL simulations.

\section{METHODS}

Beam conditioning requires a correlation between energy and transverse action. Conditioning can be obtained from a standard distribution by increasing the relative energy offset of individual electrons by an amount $\kappa J$, where $J$ is the transverse geometric action and $\kappa$ is the "conditioning parameter". FEL performance is optimized when $\kappa \simeq \lambda_{u} / 2 \lambda_{r} \beta$, where $\lambda_{u}$ is the undulator period, $\lambda_{r}$ is the output wavelength, and $\beta$ is the average beta function in the FEL. For LCLS parameters, the corresponding value is $3.2 \mu \mathrm{m}^{-1}$ which implies that a typical electron has its energy increased by $4 \mathrm{MeV}$ compared to a zero-amplitude electron (taking the emittance to be $1.2 \mu \mathrm{m}$ ). When the conditioning parameter has this optimized value, electrons with different transverse amplitudes have roughly the same parallel velocity, which helps to preserve microbunches formed in the FEL and enhances the FEL process.

One method previously considered [4] to produce a conditioned beam is to use the increased path length traversed by high-amplitude particles to delay these particles in proportion to the amplitude of their betatron motion. By imposing a large energy chirp on the beam prior to the beamline, then canceling out the chirp at the end, the main energy offset remaining should be proportional to the delay caused by path-length differences. Typically, such a design requires long propagation distances and a great deal of RF power. In this paper, we consider the use of a plasma channel to achieve very strong transverse focusing. The resulting large angles in the beam lead to rapid shifts in time of high-amplitude particles. We also consider a compact scheme where the energy chirps are generated by a plasma acceleration channel. Because of the very short wavelength of the accelerating field, the corresponding chirp can be 
achieved at a lower total voltage. The accelerating field has been treated as an idealized sinusoidal acceleration with longitudinal position, while the plasma channel is assumed to produce a simple electrostatic potential which is quadratic in radius. Although it might be expected that the focusing in the plasma channel will be perturbed by plasma oscillations and wakefields, such effects have been ignored. As a possible consequences of these effects, the usable portion of the electron beam might be reduced by a moderate factor. More technical issues such as matching the electron beam into and out of the plasma channel, which has an extremely low beta function, or also left for future work.

After the beam is conditioned, it must be accelerated to the final energy and, in some cases, compressed, without destroying the correlation between amplitude and energy. In particular, if longitudinal phase space is rotated by $90 \mathrm{de}-$ grees, the energy-amplitude correlation will be transformed into a time-amplitude correlation which does not improve FEL performance. Instead, the bunch compression must be carefully designed so that the final time coordinate is a function primarily of its time coordinate upon leaving the conditioning section, and similarly for its final energy coordinate. If designed in this way, bunch compression has the added benefit of multiplying the conditioning parameter by the compression factor, because the correlated energy shift increases by the same factor as the stochastic energy spread.

\section{NUMERICAL RESULTS}

We consider an electron beam having an energy of $1 \mathrm{GeV}$, a beam length of 20 microns and a beam emittance of 1.2 microns. These parameters are roughly those appropriate to the LCLS. The plasma channel parameters are given in Table 1. The plasma channel has a length of 10 meters and a density of $10^{16}$ particles $/ \mathrm{cm}^{3}$, which yields a matched beta function of $0.3 \mathrm{~cm}$. For this example, we consider plasma acceleration as the source of the energy chirp, and choose parameters of 300 microns for the acceleration wavelength and $100 \mathrm{MeV}$ for the total voltage, producing a chirp of $42 \mathrm{MeV}$ over 20 microns. The result is shown in Fig. 1, where energy is plotted against transverse action. The amount of conditioning is given by $\Delta \gamma / \gamma J=3.2 \mu \mathrm{m}^{-1}$. This amount would be adequate for the LCLS. We note that the beam conditioning process has a negligible effect on the magnitude of the transverse action, thus preserving emittance.

For the second example, we take the beam energy as $100 \mathrm{MeV}$, the beam length as $1 \mathrm{~mm}$ and other parameters unchanged. In this case we take the plasma channel as 2 meters long and the same plasma density of $10^{16}$ particles $/ \mathrm{cm}^{3}$, which yields a matched beta function of 0.1 $\mathrm{cm}$ for this lower beam energy. The beam chirp is generated by conventional RF, having a $10 \mathrm{~cm}$ wavelength and a total voltage of $100 \mathrm{MeV}$, producing a chirp of $6 \mathrm{MeV}$ over $1 \mathrm{~mm}$. Results are shown in Fig. 2. In this case, the amount of conditioning is $\Delta \gamma / \gamma J=0.063 \mu \mathrm{m}^{-1}$. This amount
Table 1: Conditioning plama channel parameters

\begin{tabular}{|l|l|l|}
\hline & High Energy & Low Energy \\
\hline Beam energy & $1 \mathrm{GeV}$ & $100 \mathrm{MeV}$ \\
Total accel. voltage & $100 \mathrm{MeV}$ & $100 \mathrm{MeV}$ \\
Accel. wavelength & $300 \mu \mathrm{m}$ & $10 \mathrm{~cm}$ \\
Accel. gradient & $2 \mathrm{MeV} / \mu \mathrm{m}$ & $6 \mathrm{MeV} / \mathrm{mm}$ \\
Plasma density & $10^{16} \mathrm{~cm}^{-3}$ & $10^{16} \mathrm{~cm}^{-3}$ \\
Channel length & $10 \mathrm{~m}$ & $2 \mathrm{~m}$ \\
Beta function & $0.3 \mathrm{~cm}$ & $0.1 \mathrm{~cm}$ \\
\hline
\end{tabular}

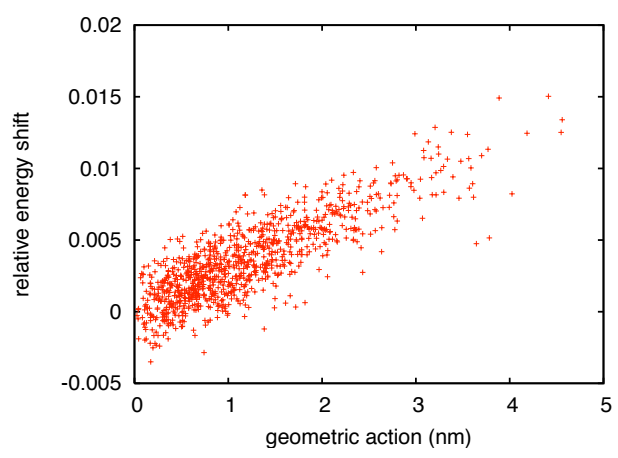

Figure 1: Correlation of energy and transverse action at the end of the plasma conditioner for a $1 \mathrm{GeV}$ beam.

would be adequate for the LCLS, assuming that subsequent beam compression amplifies the conditioning in proportion with the reduction in bunch length from $1 \mathrm{~mm}$ to $20 \mu \mathrm{m}$.

\section{NUMERICAL STUDY OF THE FEL}

For the two cases given above, we have run the resulting conditioned beams through the FEL code GENESIS [8], after accelerating it to $14 \mathrm{GeV}$ and compressing as needed. For comparison, we have also presented the case of the "standard" LCLS beam; namely, a beam at $14 \mathrm{GeV}$ with no conditioning, 3400 A peak current, 20 micron bunch length, $1.2 \mu \mathrm{m}$ emittance, and $1 \times 10^{-4}$ relative energy spread.

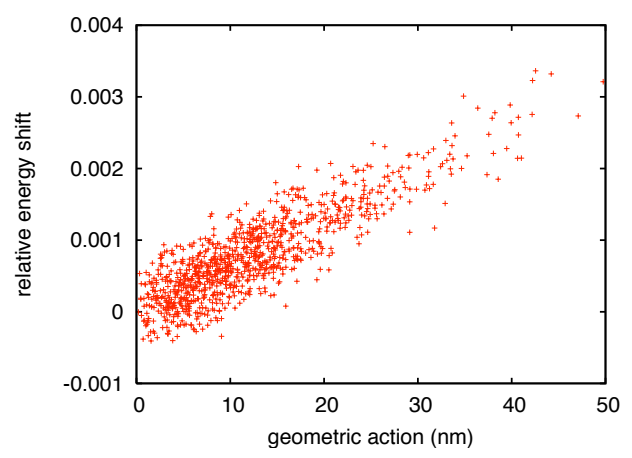

Figure 2: Correlation of energy and transverse action at the end of the plasma conditioner for a $100 \mathrm{MeV}$ beam. 
In Fig. 3, the average power is shown as a function of propagation distance when the beam has the nominal emittance. Results are given for an unconditioned beam, the beam conditioned at $100 \mathrm{MeV}$, and the beam conditioned at $1 \mathrm{GeV}$. In Fig. 4, sample output at $100 \mathrm{~m}$ is compared for these same three examples. The difference is not significant for these parameters.

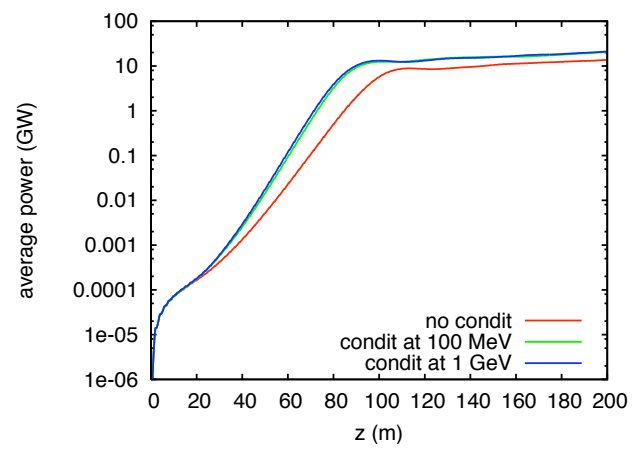

Figure 3: Average power as a function of distance for the nominal emittance beam. Three examples are shown, for unconditioned and two conditioned beams.

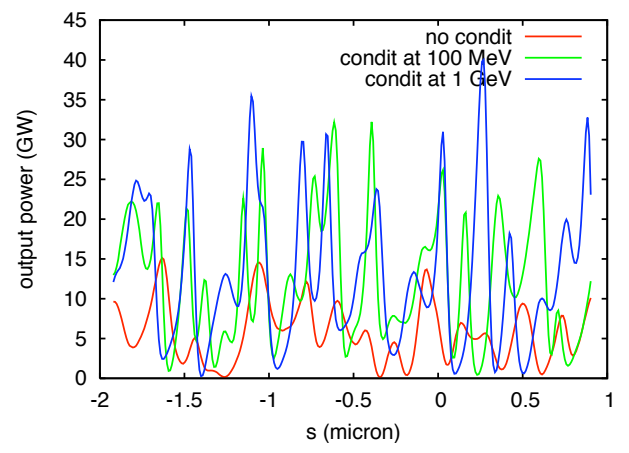

Figure 4: A sample power profile for a section near the center of the bunch, for the nominal emittance case. Three examples are shown, for unconditioned and two conditioned beams.

In Fig. 5, the average power is shown as a function of propagation distance when the beam has twice the nominal emittance. Results are given for an unconditioned beam, the beam conditioned at $100 \mathrm{MeV}$, and the beam conditioned at $1 \mathrm{GeV}$. In Fig. 6, sample output at $100 \mathrm{~m}$ is compared for these three examples. The conditioned beams are barely affected by the greater emittance, while the unconditioned beam requires significantly more undulator length to reach saturation.

\section{CONCLUSIONS}

In this paper, we have considered a plasma channel beam conditioner and shown that the concept would result in a convenient device. This study emphasizes the potential of plasma conditioners to impact future light sources, but

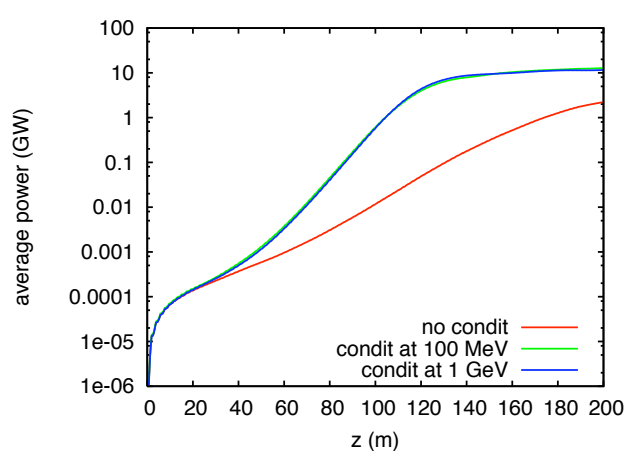

Figure 5: Average power as a function of distance for the case where the emittance is double the nominal value. Three examples are shown, for unconditioned and two conditioned beams.

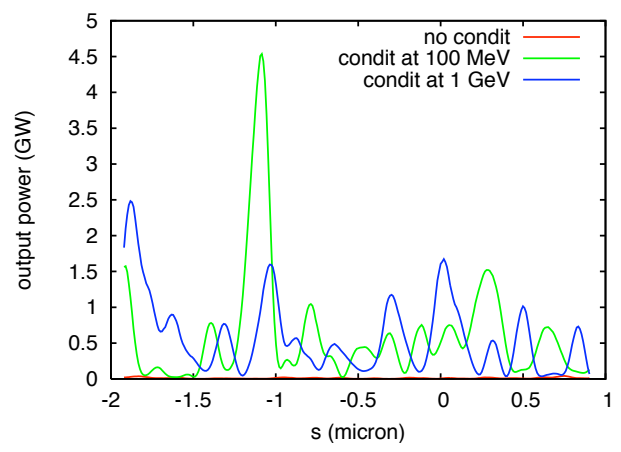

Figure 6: A sample power profile for a section near the center of the bunch, for the case where the emittance is double the nominal value. Three examples are shown, for unconditioned and two conditioned beams.

does not address important issues such as matching into the plasma structure and the method for creating the plasma focusing and acceleration. We believe that a plasma conditioner could be one of the very first applications of plasmas to conventional accelerators.

\section{REFERENCES}

[1] A.M. Sessler, D.H. Whittum, and L.-H. Yu, Phys. Rev. Lett. 68, 309 (1992).

[2] P. Emma and G. Stupakov, Phys. Rev. ST Accel. Beams 6, 030701 (2003).

[3] C.B. Schroeder, E. Esarey, and W.P. Leemans, Phys. Rev. Lett. 93, 194801 (2004).

[4] A. Wolski, G. Penn, A. Sessler, and J.S. Wurtele, Phys. Rev. ST Accel. Beams 7, 080701 (2004).

[5] A.A. Zholents, Phys. Rev. ST Accel. Beams 8, 050701 (2005).

[6] I. Blumenfeld et al., Nature 445, 6741 (2007).

[7] LCLS Design Study Group, SLAC Report No. SLAC-R-521 (1998).

[8] S. Reiche, "GENESIS 1.3: a fully 3D time-dependent FEL simulation code", Nucl. Instr. Methods A 429, 243-248 (1999). 